\title{
Editorial
}

\section{Validation of Pooled SARS-CoV-2 Testing in the Outbreak Setting}

\author{
Jessica Briggs* \\ Department of Medicine, University of California San Francisco, San Francisco, California
}

Pooled testing of specimens was first proposed in the 1940s to surveil soldiers for syphilis. ${ }^{1}$ Since then, it has been successfully used to screen donated blood for hepatitis viruses and HIV, and by public health departments to surveil for HIV and avian flu. ${ }^{2-4}$ In pooled testing, multiple samples are mixed together, and the pooled sample is assayed with a diagnostic test. If the pooled test result is negative, all individual samples that make up the pool are presumed to be negative. If the test result is positive, all of the samples in the pool are retested individually. As long as the prevalence of the targeted disease is low and most pools do not need to be retested, this strategy can massively reduce the time and resources necessary to test a large number of samples. This is an important consideration in the setting of COVID-19, where up to $50 \%$ of transmission may be from asymptomatic individuals. ${ }^{5}$ Given the worldwide shortages of laboratory reagents and test kits, pooled COVID-19 testing strategies have become especially attractive in two settings: 1) repeated surveillance of highly exposed groups, such as healthcare workers, teachers, or university students, and 2) outbreaks in countries with low prevalence of COVID-19.

Multiple groups have studied pooled testing for SARS-CoV2. A chief concern with pooling strategies is decreased sensitivity to detect the virus because of the dilution that occurs when pooling multiple samples. Usually, pooling occurs after extraction of RNA from samples. Yelin et al. ${ }^{6}$ tested known positive samples alone and combined with pools of different sizes, and found that one positive sample was consistently detected in pools of up to 32 samples; in a pool of 16 samples, sensitivity was $96 \%$. However, pooling after RNA extraction does not reduce the use of RNA extraction reagents, and so their team also attempted pooling of samples before RNA extraction. With this strategy, all positive samples were detected in pools of up to eight samples. Although they and others found that qPCR CT values increased because of decreased RNA concentration with dilution, these pooled assays are clearly sensitive enough for use in public health settings after clinical validation. ${ }^{6,7}$ At Duke University, a pooled strategy tested 10,265 students 68,913 times, diagnosing 84 , half of whom were asymptomatic, with SARS-CoV-2 infection. ${ }^{8}$ UK universities are likewise screening their student populations using pooled testing strategies. ${ }^{9}$ China used pooled testing to screen the entire population of Wuhan for SARSCoV-2 infection after an outbreak was detected in May 2020. ${ }^{10}$ In Uruguay, pooled testing has successfully been used in outbreak settings. ${ }^{11}$ Rwanda uses pooled testing to screen all passengers on incoming flights on arrival, and the government plans to use pooled testing to screen high-risk populations such as teachers and students. ${ }^{12}$

*Address correspondence to Jessica Briggs, Department of Medicine, University of California San Francisco, SFGH, Building 3, Rm 525, 1001 Potrero Ave., San Francisco, CA 94110. E-mail: jessica.briggs@ucsf.edu
However, detailed published data on the pragmatic use of pooled testing in outbreaks in resource-limited settings are lacking. Therefore, the study published in this issue of AJTMH by Thanh et al. ${ }^{13}$ is particularly timely. After 100 days without community transmission in Vietnam, two individuals with COVID-19 disease were admitted to two different hospitals in Da Nang, triggering a massive outbreak investigation. Contact tracing and testing detected cases directly linked with index cases at Da Nang Hospital. In parallel, the city was locked down on July 28,2020 , and mass community testing using a pooling strategy was performed to target those at greatest risk of infection. The study team's unique approach involved pooling all nasopharyngeal swab samples from each household (2-7 swabs per household) into viral transport medium. If a pool was positive, each household member was retested within 48 hours; 96,123 asymptomatic people met the criteria for testing, resulting in 22,290 pooled samples, of which only 24 were positive, resulting in the retesting of 104 individuals. This approach led to the identification of 32 confirmed cases, of which 22 had no history of contact with a confirmed case and would have been missed by standard contact tracing. By September 11, 2020, the city had gone 12 consecutive days without community transmission, representing a massive success in controlling the nascent outbreak. Importantly, the testing strategy required only 14 days compared with a predicted 64 days with individual testing, and allowed for a $77 \%$ cost reduction compared with testing every sample individually. In addition, pooling swabs into media (instead of pooling media from individual swabs or after RNA extraction) saved a substantial amount of freezer space, an often overlooked consideration in low-resource settings. In addition, there was no difference in the CT values of positive pooled versus positive individual samples.

Two other groups have published their experience with implementation of pooled testing for SARS-CoV-2 at a large scale. In Israel, pooled testing of samples before RNA extraction was highly efficacious despite community prevalence fluctuating between $0.5 \%$ and $6 \%$, and cost savings of $76 \%$ were realized. ${ }^{14}$ Notably, their method involved dynamically decreasing the pool size as prevalence rose in the community, which increased logistical challenges but improved efficiency. In India, a pooled testing strategy was piloted during an outbreak; pools of five samples were created before RNA extraction. ${ }^{15}$ Although a cost reduction of $68 \%$ was noted, single positives (all with high CT values, i.e., low viral loads) in four of 109 pools were missed.

Overall, there is increasing evidence for the efficacy of pooled testing strategies for the detection of SARS-CoV-2 infection in asymptomatic individuals. The study by Thanh et al. $^{13}$ published in this issue clearly shows the utility of pooled testing in a low-resource setting to screen a large asymptomatic population and prevent the expansion of a COVID-19 outbreak. Notably, this success was in conjunction 
with other aspects of a strong public health response, including a city-wide lockdown and rigorous contact tracing. This is a quite different situation than in many higher resource countries, where transmission remains high and pooling samples may not be cost-effective. However, pooled testing is likely to become more attractive in other settings as vaccination and other factors drive down community transmission. There is now sufficient evidence that pooled testing for SARSCoV-2 is sensitive enough to detect individual positive samples and mounting evidence that using this testing strategy in outbreak settings can be highly effective in detecting asymptomatic infections and reducing costs. We should look to the practical experience and success of those in lowresource settings as we consider expanding pooled testing for SARS-CoV-2 infection in the United States and other highresource settings.

Received February 16, 2021. Accepted for publication February 18, 2021.

Published online March 8, 2021.

Author's address: Jessica Briggs, Department of Medicine, University of California San Francisco, San Francisco, CA, E-mail: jessica. briggs@ucsf.edu.

This is an open-access article distributed under the terms of the Creative Commons Attribution (CC-BY) License, which permits unrestricted use, distribution, and reproduction in any medium, provided the original author and source are credited.

\section{REFERENCES}

1. Dorfman R, 1943. The detection of defective members of large populations. Annals Math Stat 14: 436-440.

2. Nguyen NT, Aprahamian H, Bish EK, Bish DR, 2019. A methodology for deriving the sensitivity of pooled testing, based on viral load progression and pooling dilution. J Transl Med 17: 252.
3. Litvak E, Tu XM, Pagano M, 1994. Screening for the presence of a disease by pooling sera samples. J Am Stat Assoc 89: 424-434.

4. Arnold ME, Slomka MJ, Coward VJ, Mahmood S, Raleigh PJ, Brown IH, 2013. Evaluation of the pooling of swabs for real-time PCR detection of low titre shedding of low pathogenicity avian influenza in Turkeys. Epidemiol Infect 141: 1286-1297.

5. Johansson MA, Quandelacy TM, Kada S, Prasad PV, Steele M, Brooks JT, Slayton RB, Biggerstaff M, Butler JC, 2021. SARSCoV-2 transmission from people without COVID-19 symptoms. JAMA Netw Open 4: e2035057.

6. Yelin I et al., 2020. Evaluation of COVID-19 RT-qPCR test in multi sample pools. Clin Infect Dis 71: 2073-2078.

7. Abdalhamid B, Bilder CR, McCutchen EL, Hinrichs SH, Koepsell SA, Iwen PC, 2020. Assessment of specimen pooling to conserve SARS CoV-2 testing resources. Am J Clin Pathol 153: 715-718.

8. Denny TN, 2020. Implementation of a pooled surveillance testing program for asymptomatic SARS-CoV-2 infections on a college campus - Duke University, Durham, North Carolina, August 2-October 11, 2020. MMWR Morb Mortal Wkly Rep 69: 1743-1747.

9. Mahase E, 2020. COVID-19: universities roll out pooled testing of students in bid to keep campuses open. BMJ 370: m3789.

10. Wee SL, Wang V, 2021. Here's how Wuhan tested 6.5 million for coronavirus in days. The New York Times, 2020 May 27; Sect. A: p. 5. Available at: https://www.nytimes.com/2020/05/26/ world/asia/coronavirus-wuhan-tests.html.

11. Taylor L, 2020. Uruguay is winning against COVID-19. This is how. BMJ. 370: m3575.

12. Mutesa $L$ et al., 2021. A pooled testing strategy for identifying SARS-CoV-2 at low prevalence. Nature 589: 276-280.

13. Thanh $T T$ et al., 2021. The application of sample pooling for mass screening of SARS-CoV-2 in an outbreak of COVID-19 in Vietnam. Am J Trop Med Hyg 104: 1530-1533.

14. Barak $\mathrm{N}$ et al.; Team THU-HC-19 Diagnosis, 2020. Lessons from applied large-scale pooling of 133,816 SARS-CoV-2 RT-PCR tests. medRxiv. 2020.10.16.20213405.

15. Singh AK et al., 2020. Evaluation of pooled sample analysis strategy in expediting case detection in areas with emerging outbreaks of COVID-19: a pilot study. PLoS One 15: e0239492. 